\title{
A Near-Capacity Differentially Encoded Non-Coherent Adaptive Multiple-Symbol-Detection Aided Three-Stage Coded Scheme
}

\author{
Li Wang, Lingkun Kong, Soon Xin $\mathrm{Ng}$ and Lajos Hanzo \\ School of ECS, University of Southampton, SO17 1BJ, UK. \\ Tel: +44-23-8059 3125, Fax: +44-23-8059 4508 \\ Email: $\{1 w 05 r, 1 k 06 r$, sxn, lh\}@ecs.soton.ac.uk; http://www-mobile.ecs.soton.ac.uk
}

\begin{abstract}
This paper presents an Irregular Distributed Hybrid Concatenated Differential (Ir-DHCD) coding scheme contrived for the relayaided differential decode-and-forward (DDF) cooperative system using multiple-symbol differential sphere detection (MSDSD), where no channel estimation is required. We proposed a practical design framework for a cooperative system, which is capable of performing close to the network's corresponding non-coherent Discrete-input Continuous-output Memoryless Channel (DCMC) capacity. An adaptive-window-duration based MSDSD scheme is employed to further reduce the iterative detection complexity. Specifically, upon using the proposed near-capacity system design, the Ir-DHCD coding scheme devised becomes capable of performing within about $1.8 \mathrm{~dB}$ from the corresponding single-relayaided DDF cooperative system's DCMC capacity.
\end{abstract}

\section{INTRODUCTION}

Multiple antenna aided diversity techniques $[1,2]$ constitute powerful arrangements of mitigating the deleterious effects of fading, hence improving the end-to-end system performance, which is usually achieved by multiple co-located antenna elements at the transmitter and/or receiver. However, it is often impractical for the mobile to employ a large number of antennas for the sake of achieving a diversity gain due to its limited size. Fortunately, in multi-user wireless systems cooperating mobiles may share their antennas in order to achieve uplink transmit diversity by formig a virtual antenna array (VAA) in a distributed fashion [2,3]. On the other hand, in order to avoid channel estimation for a VAA-aided system, which may impose both an excessive complexity and a high pilot overhead, especially in mobile environments associated with relatively rapidly fluctuating channel conditions, differentially encoded transmissions [2] combined with non-coherent detection requiring no channel state information (CSI) at the receiver becomes an attractive design alternative, leading to differential modulation assisted cooperative communications [4-6].

Inspired by the classic turbo codes used in non-cooperative communication scenarios, distributed turbo codes [7] have been proposed for "distributed MIMO" systems, which benefit from a turbo processing gain. Furthermore, in order to mitigate the detrimental impact of the above-mentioned time-selective channel induced by the relative mobility amongst transceivers, multiple-symbol differential sphere detection (MSDSD) has been recently proposed in [8] and has been further developed to be implemented in the differentially modulated cooperative system in [5].

Against this background, our objective in this paper is to propose a practical framework of designing a cooperative system, which is capable of performing close to the network's corresponding non-coherent Discrete-input Continuous-output Memoryless Channel (DCMC) [9, 10] capacity. Based on our low-complexity near-capacity design criterion, a novel Irregular Distributed Hybrid Concatenated Differential (Ir-DHCD) coding scheme is proposed for the differential decode-and-forward (DDF) cooperative system [6] employing the

Acknowledgements: The finacial support of the EPSRC UK under the auspices of the UK-India centre of Excellence in Wireless Communications and that of the EU Optimix project is gratefully acknowledged. proposed capacity-achieving low-complexity adaptive-window-aided soft-input soft-output (SISO) iterative MSDSD scheme.

\section{SYSTEM MODEL}

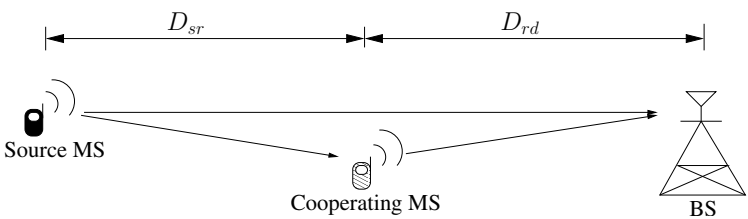

Fig. 1. Single-relay-aided cooperative cellular uplink.

The TDMA-based DDF-aided cooperative cellular uplink [6] is considered without any loss of generality, where no CSI estimation is required. For the sake of simplicity, we consider the singlerelay-assisted scenario of Figure 1, where only one coopearting mobile station (MS) is activated in order to decode and re-encode the signal received from the source MS prior to forwarding the signal to the base station (BS). The employment of a single antenna per terminal is assumed, owing to the cost- and size-constraints of portable transceivers. A general cooperative scenario is created here by simply assuming that the total transmit power is equally divided between the source MS and the single cooperating RS, which is located half-way between the source MS and the BS, as depicted in Figure 1. More specifically, for the sake of analytical tractability, we simply assume that the sum of the normalized distances $D_{s r}$ between the source MS and the RS, and that between the RS and the $\mathrm{BS}$, which is represented by $D_{r d}$, is equal to the normalized distance $D_{s d}$ between the source MS and the BS. Naturally, the normalized SD-distance is equal to unity. As a result, observe in Figure 1 that we have: $D_{s r}=D_{r d}=\frac{1}{2} D_{s d}=0.5$. Furthermore, the normalized average power $\sigma_{i, j}^{2}$ at the output of the channel is inversely proportional to the inter-node distance $D_{i, j}$, which may be rewritten as $\sigma_{i, j}^{2}=D_{i, j}^{-v},(i, j \in\{s, r, d\})$, where $v$ denotes the path-loss exponent [9] and the subscripts $s, r$ and $d$ represent the source, relay and destination, respectively.

Throughout this paper a time-selective block-fading Rayleigh channel is assumed, where the fading coefficients are assumed to change in an independent and identically-distributed (i.i.d.) manner from block to block, but the channel's envelope exhibits correlation within each transmission block according to the normalized Doppler frequency $f_{d}$ induced by the relative movement of the tranceivers.

Consider a single-antenna-assisted point-to-point transmission scheme communicating over a the above-mentioned block-fading channel, which exhibits a correlated envelope for the duration of $T_{b}$ consecutive symbols. Then, the received signal may be formulated as:

$$
\mathbf{y}=\mathbf{S}_{d} \mathbf{h}+\mathbf{w}
$$

where we have $\mathbf{y}=\left[y_{1}, y_{2}, \cdots, y_{T_{b}}\right]^{T}, \mathbf{h}=\left[h_{1}, h_{2}, \cdots, h_{T_{b}}\right]^{T}$, and $\mathbf{w}=\left[w_{1}, w_{2}, \cdots, w_{T_{b}}\right]^{T}$ representing the received signal's 
column vector, the fading coefficient's column vector obeying a complex-valued Gaussian distribution $\mathcal{C N}\left(0, \sigma_{h}^{2}\right)$ and the Gaussian noise column vector having a distribution of $\mathcal{C} \mathcal{N}\left(0,2 \sigma_{w}^{2}\right)$, respectively. The diagonal matrix $\mathbf{S}_{d}$ in Eq.(1) hosts the $T_{b}$ consecutively transmitted signals within a block along its diagonal, which may be expressed as $\mathbf{S}_{d}=\operatorname{diag}\{\mathbf{s}\}$, where we have $\mathbf{s}=\left[s_{1}, s_{2}, \cdots, s_{T_{b}}\right]^{T}$.

\section{CAPACITy-Achieving Multiple-Symbol DifFEREnTial SPHERE DETECTION}

\section{A. Soft-Input Soft-Output MSDSD}

Let us now briefly review the SISO MSDSD scheme, which will be used in our forthcoming near-capacity design for the DDF-aided cooperative system. The PDF of the non-coherently received signal's vector $\mathbf{y}$ in Eq. (1) was conditioned on the transmitted signal vector s, which may be readily expressed for Rayleigh fading channels as [11]:

$$
p(\mathbf{y} \mid \mathbf{s})=\frac{\exp \left(-\mathbf{y}^{H} \Psi^{-1} \mathbf{y}\right)}{\operatorname{det}(\pi \Psi)},
$$

where we have $\Psi=\mathcal{E}\left\{\mathbf{y} \mathbf{y}^{H} \mid \mathbf{s}\right\}=\mathbf{S}_{d} \Sigma_{h} \mathbf{S}_{d}^{H}+2 \sigma_{w}^{2} \mathbf{I}_{T_{b}}$ with $\Sigma_{h}$ denoting the channel's covariance matrix. Therefore, the a posteriori Log-Likelihood-Ratio (LLR) of the $k$ th transmitted bit $x_{k}$ at the output of the maximum-a-posterori multiple-symbol differential detector (MAP-MSDD) [8], which jointly and differentially detects $\left(N_{\text {wind }}-1\right)$ data symbols, can be evaluated with the aid of Bayes' theorem as [12]:

$$
\begin{aligned}
L_{D 1}\left(x_{k}\right) & =\ln \frac{\operatorname{Pr}\left(x_{k}=+1 \mid \mathbf{y}\right)}{\operatorname{Pr}\left(x_{k}=-1 \mid \mathbf{y}\right)} \\
& =\ln \frac{\sum_{\mathbf{x} \in \mathbb{X}_{k,+1}} p(\mathbf{y} \mid \mathbf{x}) \operatorname{Pr}(\mathbf{x})}{\sum_{\mathbf{x} \in \mathbb{X}_{k,-1}} p(\mathbf{y} \mid \mathbf{x}) \operatorname{Pr}(\mathbf{x})} \\
& =\ln \frac{\sum_{\mathbf{x} \in \mathbb{X}_{k,+1}} \exp \left[-\mathbf{y}^{H} \Psi \mathbf{y}+\ln (\operatorname{Pr}(\mathbf{x}))\right]}{\sum_{\mathbf{x} \in \mathbb{X}_{k,-1}} \exp \left[-\mathbf{y}^{H} \Psi \mathbf{y}+\ln (\operatorname{Pr}(\mathbf{x}))\right]},
\end{aligned}
$$

where $\mathbb{X}_{k,+1}$ represents the set of $\frac{M_{c}^{\left(N_{\text {wind }}-1\right)}}{2}$ number of legitimate transmitted bit vectors $\mathbf{x}$ associated with $x_{k}=+1$, and similarly, $\mathbb{X}_{k,-1}$ is defined as the set corresponding to $x_{k}=-1$. Furthmore, $\operatorname{Pr}(\mathbf{x})$ of Eq. (5) is the a priori probability, which may be computed based on the a priori LLRs delivered by the outer channel decoder. It was shown in [12] that after a few mathematical manipulations and with the aid of the "sum-max" approximation, a reduced-complexity computation of the a posteriori LLRs may be formulated as:

$$
\begin{aligned}
L_{D 1}\left(x_{k}\right) \approx & -\left\|\mathbf{U} \hat{\mathbf{s}}_{M A P}^{x_{k}=+1}\right\|^{2}+\ln \left[\operatorname{Pr}\left(\hat{\mathbf{x}}_{M A P}^{x_{k}=+1}\right)\right] \\
& +\left\|\mathbf{U} \hat{\mathbf{s}}_{M A P}^{x_{k}=-1}\right\|^{2}-\ln \left[\operatorname{Pr}\left(\hat{\mathbf{x}}_{M A P}^{x_{k}=-1}\right)\right],
\end{aligned}
$$

where $\mathbf{U}$ is an upper-triangular matrix, which can be obtained as $\mathbf{U} \triangleq$ $(\mathbf{F} \operatorname{diag}\{\mathbf{y}\})^{*}$, where $\mathbf{F}$ is also an upper-triangular matrix generated using the Cholesky factorization of the matrix $\left(\Sigma_{h}+2 \sigma_{w}^{2} \mathbf{I}_{N_{\text {wind }}}\right)^{-1}$. Consequently, thanks to the upper-triangular structure of the matrix $\mathbf{U}$, when evaluating Eq. (6), we arrive at $\hat{\mathbf{s}}_{M A P}^{x_{k}=b}$ and $\hat{\mathbf{x}}_{M A P}^{x_{k}=b}$, which represent the MAP-algorithm-based symbol vector estimate and the MAP-based bit vector estimate, respectively, that may be obtained with the aid of the low-complexity sphere detection (SD) algorithm of [12] by fixing the $k$ th bit value to $b,(b=-1$ or +1$)$.

In the sequel, the extrinsic LLR, $L_{E 1}\left(x_{k}\right)$ may be obtained by excluding the corresponding a priori LLR, $L_{A 1}\left(x_{k}\right)$, from the $a$ posteriori LLR, $L_{D 1}\left(x_{k}\right)$, according to $L_{E 1}\left(x_{k}\right)=L_{D 1}\left(x_{k}\right)-$ $L_{A 1}\left(x_{k}\right)$, which is then exploited by the outer decoder after passing it through the interleaver.

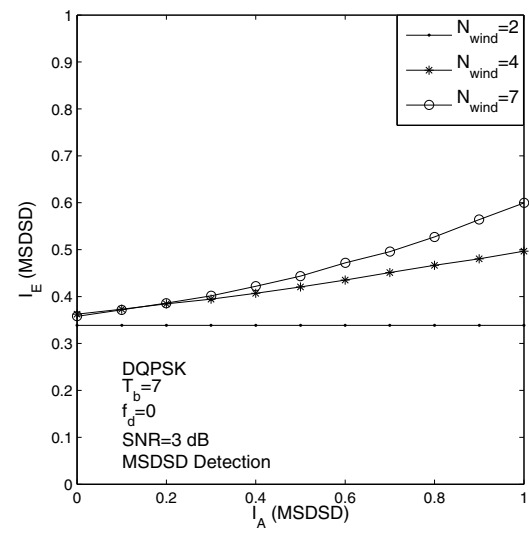

Fig. 2. EXIT curves of the MSDSD for various values of $N_{\text {wind }}$.

In order to visualize the Extrinsic Information Transfer (EXIT) charateristics [13] of the SISO MSDSD scheme, we plot the EXIT curves associated with different observation window sizes of $N_{\text {wind }}$ in Figure 2 by evaluating the extrinsic mutual information, $I_{E}$, at the output of the MSDSD for a given input stream of bit LLRs along with the a priori mutual information $I_{A}$ at $\mathrm{SNR}$ of $3 \mathrm{~dB}$ in the scenario of a conventional point-to-point system. As observed in Figure 2, significantly increased iterative gains may be achieved when jointly and differentially detecting $\left(N_{\text {wind }}-1\right)>1$ data symbols using the MSDSD.

\section{B. Maximum Achievable Rate versus the Capacity: An EXIT Chart Perspective}

According to the area properties of EXIT charts [13], the area $\mathcal{A}$ under the bit-based EXIT curve of a soft-detector/soft-demapper is equal to the maximum possible code rate $R_{\text {outer,max }}$ of the outer channel code that can be employed to achieve near errorfree transmissions. Hence, the maximum achievable near-error-free transmission rate $R_{\text {overall,max }}$ of a differentially encoded system is computed as:

$$
R_{\text {overall,max }}=\left(\frac{T_{b}-1}{T_{b}} \log _{2} M_{c}\right) \cdot \mathcal{A} \quad \text { bits } / \mathrm{s} / \mathrm{Hz}
$$

which may be improved with the aid of the MSDSD. The ratio of $\frac{T_{b}-1}{T_{b}}$ accounts for the rate-loss induced by the reference signal transmission in the classic differential signalling process.

In the sequel, the maximum achievable rate of a differentially encoded system employing the MSDSD may be plotted against the SNR, as shown in Figure 3(a), by evaluating the area under the corresponding EXIT curve of the MSDSD. On the other hand, the MAP-based MSDSD employing the highest possible observation window size, namely $N_{\text {wind }}=T_{b}$, can be regarded as the optimum differential detector in the interest of approaching the theoretically maximum transmission rate for a given differentially encoded modulation scheme. Figure 3(b) depicts the maximum achievable rate curve of the system employing the MSDSD for various fading block lengths $T_{b}$, which almost coincides with the non-coherent DCMC capacity [11]. The slight gap between them is not unexpected, since the "summax" approximation algorithm of Eq. (6) is employed by the MSDSD in order to reduce the complexity imposed by the computation of the a posteriori LLRs. 

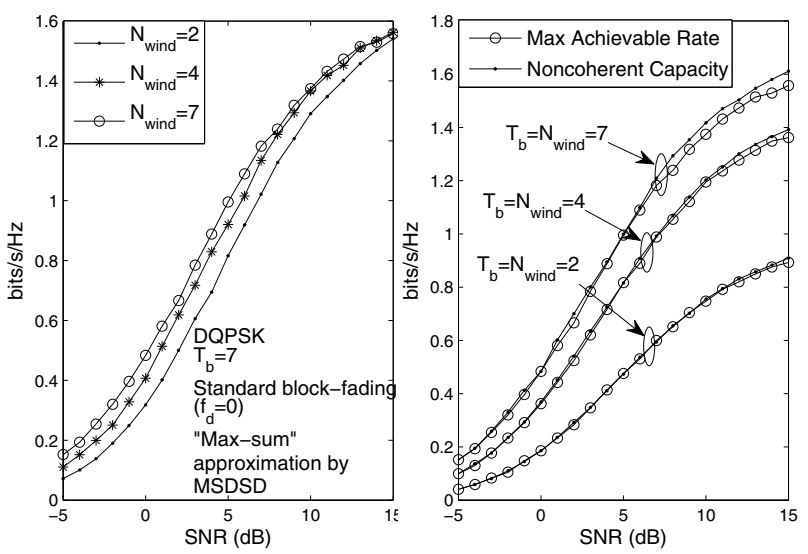

(a) Different obseration window size (b) Different fading block size

Fig. 3. Maximum achievable rate of the single-input single-output differentially encoded QPSK modulated system using the MSDSD.

\section{IRREgular Distributed Hybrid CONCATENATED DIFFERENTIAL ENCODING/DECODING}

In conventional relay-aided decode-and-forward (DF) cooperative systems, the relay decodes the signal received from the source and re-encodes it using an idential channel encoder. Then the destination receives two versions of the same code word, namely those from the source and relay, respectively, which may be viewed as a repetition code. Finally, the two replicas of the signal may be combined using maximal ratio combining (MRC) prior to the decoding. In order to enhance the coding gain achieved by the repetition code constituted by the relay-aided system, while simultaneously maintaining the cooperative diversity gain, the classic turbo coding mechanism was introduced into the DF aided cooperative system of [7], resulting in the so-called distributed turbo coding scheme. Specifically, according to the principle of parallel concatenated convolutional code based turbo coding, the data and its interleaved version are encoded in parallel, using two distinct recursive systematic convolutional (RSC) codes, respectively. Therefore, a distributed turbo code may be readily constructed at the relay by interleaving its received estimated source data prior to re-encoding. Consequently, a turbo-like decoder may be implemented at the destination. It was revealed in [7] that a significantly enhanced coding gain can be achieved by a distributed turbo code in comparison to that attained by a single convolutional code for transmission over two independently fading channels.

In order to improve the iterative decoder's achievable performance and hence achieve near-error-free transmissions between the source and relay, a unity-rate-coded (URC) three-stage serially concatenated transceiver employing so-called Irregular Convolutional Codes (Ir$\mathrm{CCs}$ ), may be employed in the single-relay-aided DDF cooperative system. More specifically, since the URC has an infinite impulse response due to its recursive encoder architecture, the resultant EXIT curve of the URC-aided inner decoder is capable of reaching the point $(1,1)$ of the EXIT chart, provided that the interleaver length is sufficiently high [13]. Furthermore, since the URC decoder employs the MAP decoding scheme, the extrinsic probability generated at the output of the URC decoder contains the same amount of information as the sequence at the input of the URC decoder [14]. In other words, the area under the inner EXIT curve remains the same, regardless of the URC's employment ${ }^{1}$. Hence, a higher ending point of the EXIT

\footnotetext{
${ }^{1}$ This statement exploits the specific property of the EXIT chart that the area under the inner decoder's EXIT curve is proportional to the system's capacity [14].
}

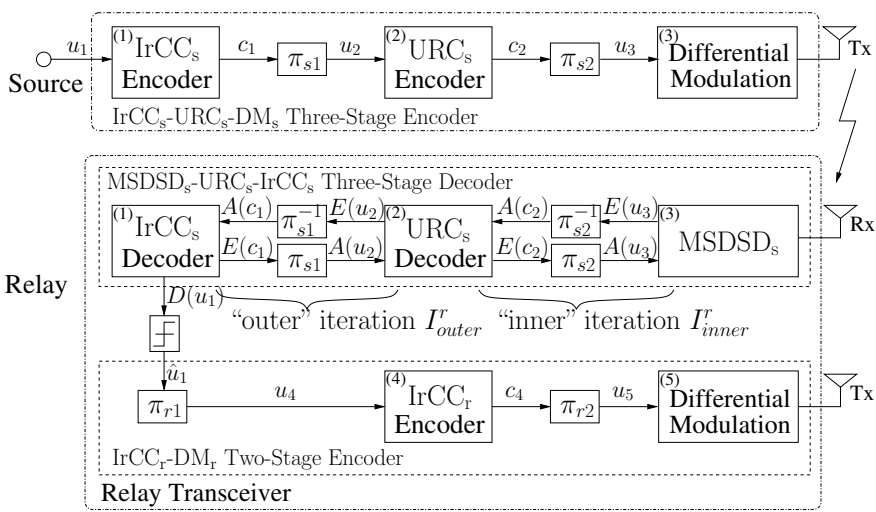

Fig. 4. Schematic of the irregular distributed hybrid concatenated differential coding encoder.

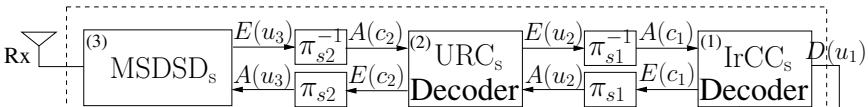

$$
\begin{aligned}
& \text { Phase I: MSDSD } \mathrm{s}_{\mathrm{s}}-\mathrm{URC}_{\mathrm{s}}-\mathrm{IrCC}_{\mathrm{s}} \text { Decoder } A\left(u_{1}\right)^{*} E\left(u_{1}\right) \\
& \text { "inner" iteration } I_{\text {inner } 1}^{d} \text { "inner" iteration } I_{\text {inner } 2}^{d} \pi_{r 1}^{-1}\left[\begin{array}{ll}
\pi_{r 1} \\
\hline
\end{array}\right.
\end{aligned}
$$

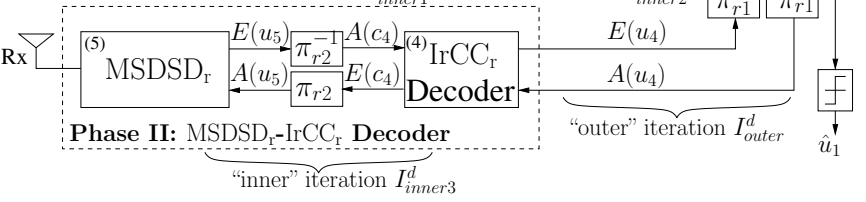

Fig. 5. Iterative receiver at the destination BS.

curve at $I_{A}=1$ leads to a lower starting point at $I_{A}=0$, implying a steeper slope for the EXIT curve, which in turn yields a reduced error floor and a higher SNR threshold [2], above which decoding convergence to a vanishingly low BER becomes possible, as we will demonstrate in Section V.

Based on the above arguments, the transmitter's architecture proposed for the source is depicted in Figure 4, where we use a conventional differential modulation scheme, such as DQPSK, which is amalgamated with the URC encoder in order to create a twostage inner code, whereas an IrCC associated with an average code rate of $R_{s}$, namely $\mathrm{IrCC}_{s}$, is employed as the outer code for the sake of achieving a performance that is close to the capacity of the SR link. The corresponding URC decoder assisted three-stage receiver proposed for the relay is also portrayed in Figure 4 together with its two-stage transmitter schematic. Specifically, at the receiver of the relay, which is constituted by three modules, namely the MSDSD $s$, the $\mathrm{URC}_{s}$ decoder and the $\mathrm{IrCC}_{s}$ decoder, the extrinsic information is exchanged amongst the modules in a number of consecutive iterations. As shown in Figure 4, $A(\cdot)$ represents the a priori information expressed in terms of the LLRs, while $E(\cdot)$ denotes the corresponding extrinsic information. At the transmitter of the relay, the estimated data bit stream is fed through the interleaver $\pi_{r 1}$ prior to the $\mathrm{IrCC}_{r}$ encoder having an average code rate of $R_{r}$, as observed in Figure 4, in order to construct a distributed turbo code together with the source. Consequently, the proposed relay-aided cooperative system may be referred to here as an Irregular Distributed Hybrid Concatenated Differential (Ir-DHCD) coding scheme, under the assumption of an error-free decoding at the relay.

At the destination BS, according to the principles of the distributed turbo decoding mechanism proposed in [7], the novel iterative receiver of Figure 5 is used for decoding the Ir-DHCD coding scheme of Figure 4. To be specific, the first part of the iterative receiver is an amalgamated " $\mathrm{MSDSD}_{s}-\mathrm{URC}_{s}-\mathrm{IrCC}_{s}$ " iterative decoder, which is used to iteratively decode the signal received directly from the source during Phase I, while the second part is constituted of the $\mathrm{MSDSD}_{r}$ 

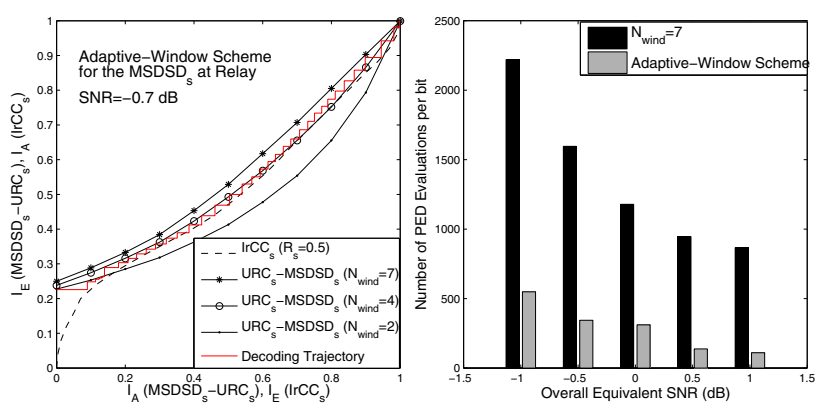

(a) EXIT trajectory at relay MS. (b) Complexity reduction achieved.

Fig. 6. Characterization of the adaptive-window aided scheme for the $\mathrm{MSDSD}_{s}$ at the relay.

differential detector and the $\mathrm{IrCC}_{r}$ decoder, which is employed to iteratively decode the signal forwarded by the relay during Phase II. Since the "MSDSD ${ }_{s}-\mathrm{URC}_{s}-\mathrm{IrCC}_{s}$ " decoder and the "MSDSD ${ }_{r}$ $\mathrm{IrCC}_{r}$ " decoder may be regarded as the two-component decoders of a turbo receiver, the extrinsic information exchange between them, which is referred to as "the "outer iteration", is expected to significantly enhance the achievable coding gain.

\section{NEAR-CAPACITY SySTEM DESIGN}

\begin{tabular}{l|r}
\hline \multicolumn{2}{c}{ Single-Relay-Aided Cooperative Cellular Uplink } \\
\hline \hline Path Loss Exponent & $v=3$ \\
\hline Doppler Frequency & $f_{d}=0.01$ \\
\hline Fading Block Size & $T=7$ \\
\hline Tx at Source or Relay MS & 1 \\
\hline Rx at Relay MS or BS & 1 \\
\hline Modulation & DQPSK \\
\hline Detector/MAP & MSDSD \\
\hline Channel Code & IrCC (17 subcodes) \\
\hline Code Rate at Source MS & $R_{s}=0.5$ \\
\hline
\end{tabular}

TABLE I

SUMMARY OF SYSTEM PARAMETERS

In this section, we propose a practical framework, which enables the proposed Ir-DHCD scheme to approach the cooperative system's capacity. Without loss of generality, the average code rate $R_{s}$ of the $\mathrm{IrCC}_{s}$ at the source is chosen to be 0.5 . All other system parameters are summarized in Table I. As it will be shown in our forthcoming discourse, the joint source-and-relay mode design procedure can be decoupled into two EXIT curve matching problems.

\section{A. Reduced-Complexity Near-Capacity Design at Relay}

Although it is not explicitly demonstrated here owing to the lack of space, through our EXIT chart analysis, the iterative information exchange between the MSDSD $s$ and $\mathrm{URC}_{s}$ blocks of the relay receiver in Figure 4 approaches convergence as early as at their second iteration. Hence, the number of iterations between the $\mathrm{MSDSD}_{s}$ and $\mathrm{URC}_{s}$ blocks was set to $I_{\text {inner }}^{r}=2$ in our future simulations, in order to avoid any unnecessarily imposed complexity. In practice, for the sake of avoiding a potentially excessive complexity at the relay, while approaching the capacity, a 'higher-than-necessary' EXIT curve associated with $N_{\text {wind }}=7$ may be ensured for the inner combined "MSDSD ${ }_{s}-\mathrm{URC}_{s}$ " decoder by having a slightly 'higherthan-necessary' overall equivalent SNR of, for example, $-0.7 \mathrm{~dB}$, as depicted in Figure 6(a). The overall equivalent $S N R^{2}, S N R_{e}^{\text {overall }}$ is

\footnotetext{
${ }^{2}$ The terminology of 'equivalent SNR' is used here to indicate the fact that it quantifies the ratio of the transmit power and the receiver's noise, which are measured at physically different points.
}

defined as the sum of the equivalent SNRs at both the source and relay transmitters, hence we have $S N R_{e}^{s}=S N R_{e}^{r}=\frac{1}{2} S N R_{e}^{\text {overall }}$ under the equal-power-allocation assumption. Then, using the EXIT curve of the inner amalgamated "MSDSD $-\mathrm{URC}_{s}$ " decoder, the optimized weighting coefficients of the half-code-rate $\mathrm{IrCC}_{s}$ can be obtained with the aid of the EXIT curve matching algorithm of [15], resulting in a narrow but marginally open tunnel between the EXIT curves of the inner amalgamated "MSDSD ${ }_{s}-\mathrm{URC}_{s}$ " decoder and the outer $\mathrm{IrCC}_{s}$ decoder, as seen in Figure 6(a).

On the other hand, in order to further reduce the complexity imposed by the "MSDSD $-\mathrm{URC}_{s}$ " decoder during the iterative decoding process at the relay, an adaptive-window-duration based scheme is proposed for the $\mathrm{MSDSD}_{s}$, where the observation window size was initially set to the smallest value of $N_{\text {wind }}=2$, which will be slightly increased, as soon as the iterative decoding process between the "MSDSD ${ }_{s}-\mathrm{URC}_{s}$ " decoder and the $\mathrm{IrCC}_{s}$ decoder converges. The proposed adaptive-window-duration based scheme is characterized by Figure 6(a). Indeed, the complexity imposed is significantly reduced by the adaptive-window-duration based scheme, as observed in Figure 6(b), where the complexity imposed by the $\mathrm{MSDSD}_{s}$ in terms of the number of the PED evaluations per bit is plotted versus the overall equivalent SNR for both systems operating with and without the adaptive-window-duration based scheme. Remarkably, the complexity imposed by the $\mathrm{MSDSD}_{s}$ is substantially reduced in Figure 6(b) with the aid of the adaptive-window-duration assisted scheme by as much as $75 \%$ at $S N R_{t}^{\text {overall }}=2 \mathrm{~dB}$, when the open EXIT tunnel created by having $N_{\text {wind }}=7$ is rather narrow. This is not unexpected, since although an increased number of iterations may be needed between the "MSDSD ${ }_{s}-\mathrm{URC}_{s}$ " decoder and the $\mathrm{IrCC}_{s}$ decoder to achieve the same amount of iteration gain, when the adaptive-window-duration based scheme is employed, the complexity per iteration imposed by the $\mathrm{MSDSD}_{s}$ using a reduced $N_{\text {wind }}$ value is expected to be exponentially reduced, yielding a potentially reduced overall complexity.

\section{B. Reduced-Complexity Near-Capacity Design at Destination}

In Section V-A, the $\mathrm{IrCC}_{s}$ decoder of Figure 4 was specifically designed to allow a near-capacity operation over the SR link with the aid of the EXIT curve matching algorithm of [15] carried out at the relay. Let us now consider the destination BS and optimize the weighting coefficients of the other irregular convolutional code, i.e. those of the $\mathrm{IrCC}_{r}$, employed by the relay's transmitter in Figure 4. Based on the above-mentioned desirable choice of $I_{\text {inner }}^{r}=2$, we also set the number of iterations between the $\operatorname{MSDSD}_{s}$ and $\mathrm{URC}_{s}$ blocks of the BS in Figure 5 to $I_{\text {inner } 1}^{d}=2$. Then, we continue by determining the desirable number of iterations between the combined "MSDSD ${ }_{s}-\mathrm{URC}_{s}$ " decoder and the $\mathrm{IrCC}_{s}$ decoder, $I_{\text {inner } 2}^{d}$, as well as that required between the $\mathrm{MSDSD}_{r}$ and the $\mathrm{URC}_{r}$ arrangements, $I_{\text {inner } 3}^{d}$, by plotting the corresponding EXIT curves of the amalgamated "MSDSD ${ }_{s}-\mathrm{URC}_{s}-\mathrm{IrCC}_{s}$ " decoder as well as those of the combined "MSDSD ${ }_{r}-\mathrm{IrCC}_{r}$ " decoder associated with different values of $I_{\text {inner } 2}^{d}$ and $I_{\text {inner } 3}^{d}$, respectively, as seen in Figure 7. Specifically, observe in Figure 7(a) that a sharply-rising EXIT curve can be created, when using our low-complexity nearcapacity design criterion, since only $I_{\text {inner } 1}^{d}=2$ iterations are required between the $\mathrm{MSDSD}_{s}$ and the $\mathrm{URC}_{s}$, while $I_{\text {inner } 2}^{d}=6$ iterations may be necessitated between the combined "MSDSD ${ }_{s}$ $\mathrm{URC}_{s}$ " decoder and the $\mathrm{IrCC}_{s}$ of Figure 5. Similarly, observe the EXIT curves of the combined "MSDSD ${ }_{r}-\mathrm{IrCC}_{r}$ " decoder associated with as few as three IrCC subcodes in Figure 7(b), which indicates that although the EXIT curve of "MSDSD ${ }_{r}-\mathrm{URC}_{r}$ " can be shifted to the left by increasing the number of iterations between the $\mathrm{MSDSD}_{r}$ 


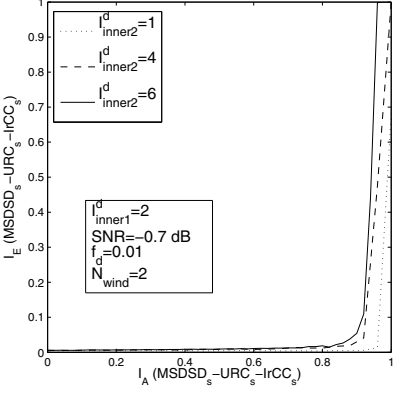

(a) "MSDSD $s-\mathrm{URC}_{s}-\mathrm{IrCC}_{s}$ "

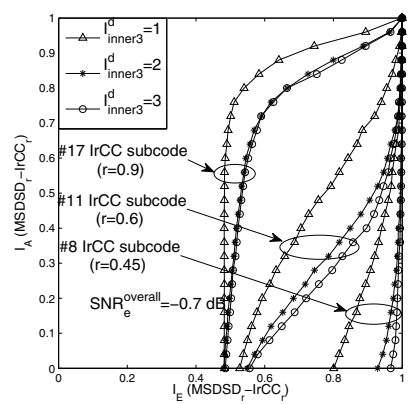

(b) "MSDSD $r-\operatorname{IrCC}_{r}$ "

Fig. 7. EXIT curves of the amalgamated "MSDSD $s-\mathrm{URC}_{s}-\mathrm{IrCC}_{s}$ " decoder and the combined "MSDSD $r-\mathrm{IrCC}_{r}$ " decoder employed by the destination BS.

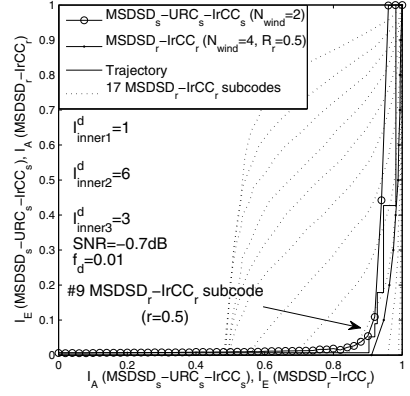

(a) Iterative decoding trajectory at the BS.

Fig. 8. The achievable performance of the near-capacity designed system.

and the $\mathrm{IrCC}_{r}$, any further shifting of the EXIT curve to the left in the interest of creating a wider EXIT tunnel is becoming rather marginal, when the number of iterations exceeds $I_{\text {inner } 3}^{d}=2$. Hence, based on the low-complexity near-capacity design criterion, the number of iterations between the $\mathrm{MSDSD}_{r}$ and the $\mathrm{IrCC}_{r}$ blocks of Figure 5 is chosen to be $I_{\text {inner } 3}^{d}=2$.

Finally, we use the EXIT curve matching algorithm of [15] in order to match the SNR-dependent EXIT curve of the combined "MSDSD ${ }_{r}-\mathrm{IrCC}_{r}$ " decoder of Figure 5 to the target EXIT curves of the amalgamated "MSDSD $-\mathrm{URC}_{s}-\mathrm{IrCC}_{s}$ " decoder of the $\mathrm{BS}$ portrayed in 7(a), as shown in Figure 8(a). As a result, for the sake of achieving a near-capacity performance, while maintaining a moderate computational complexity, a 'wider-than-necessary' EXIT tunnel is created between the EXIT curve of the amalgamated "MSDSD ${ }_{s}$ $\mathrm{URC}_{s}-\mathrm{IrCC}_{s}$ " decoder and that of the combined "MSDSD $r-\mathrm{IrCC}_{r}$ " decoder at the BS. Thus, the resultant average coding rate of the designed $\mathrm{IrCC}_{r}$ scheme is equal to $R_{r}=0.5$, which cannot be achieved by simply using one of $17 \mathrm{IrCC}_{r}$ subcodes having the same code rate, as observed in Figure 8(a), owing to the absence of an open EXIT tunnel.

\section{Simulation Results and Discussions}

We have now completed the low-complexity near-capacity system design for the single-relay-aided cooperative system of Table I. Since the average code rates are fixed to $R_{s}=R_{r}=0.5$ for both the $\operatorname{IrCC} C_{s}$ at the transmitter of the source and the $\mathrm{IrCC}_{r}$ employed at the relay, we can calculate the network's bandwidth efficiency as follows [10]:

$$
\eta^{\text {coop }}=\frac{R_{s} R_{r}}{R_{s}+R_{r}} \frac{T_{b}-1}{T_{b}} \log _{2} M_{c}=0.4286 \mathrm{bits} / \mathrm{s} / \mathrm{Hz} .
$$

The corresponding Monte-Carlo simulation based decoding trajectory is now plotted in Figure $8(\mathrm{a})$, which reaches the $(1.0,1.0)$ point of the EXIT chart, indicating the achievement of decoding convergence to an infinitesimally low BER at near-capacity SNRs for the Ir-DHCD coding scheme proposed in Section IV. In Figure 8(b), the BER curve of a 'well-designed' cooperative system is portrayed in comparison to that of the conventional near-capacity point-to-point transmission based system having an identical bandwidth efficiency. In conclusion, upon using the near-capacity system design of Section $\mathrm{V}$, the proposed Ir-DHCD coding scheme becomes capable of performing within about $1.8 \mathrm{~dB}$ from the corresponding single-relayaided DDF cooperative system's DCMC capacity [10]. Moreover, in the typical urban area cellular radio environment associated with $v=3$, the single-relay-aided cooperative system becomes capable of significantly outperforming the direct-transmission based system, requiring an overall transmit power which is about $2.5 \mathrm{~dB}$ lower than that needed by the latter to achieve an infinitesimally low BER.

\section{REFERENCES}

[1] G. J. Foschini and M. J. Gans, "On limits of wireless communications in a fading environment when using multiple antennas," Wireless Personal Communications, vol. 6, pp. 311-335, Mar. 1998.

[2] L. Hanzo, O. Alamri, M. El-Hajjar, and N. Wu, Near-Capacity MultiFunctional MIMO Systems: sphere-packing, iterative detection, and cooperation. First Edition, John Wiley \& Sons Ltd, 2009.

[3] J. N. Laneman, D. N. C. Tse, and G. W. Wornell, "Cooperative diversity in wireless networks: Efficient protocols and outage behavior," IEEE Transaction on Information Theory, vol. 50, pp. 3062-3080, Dec. 2004.

[4] T. Himsoon, W. Su, and K. J. R. Liu, "Differential transmission for amplify-and-forward cooperative communications," IEEE Signal Processing Letters, vol. 12, pp. 597-600, Sept. 2005.

[5] L. Wang and L. Hanzo, "The amplify-and-forward cooperative uplink using multiple-symbol differential sphere-detection," IEEE Signal Processing Letters, vol. 16, pp. 913-916, Oct. 2009.

[6] L. Wang and L. Hanzo, "The resource-optimized differentially modulated hybrid $\mathrm{AF} / \mathrm{DF}$ cooperative cellular uplink using multiple-symbol differential sphere detection," IEEE Signal Processing Letters, vol. 16, pp. 965-968, Nov. 2009.

[7] B. Zhao and M. C. Valenti, "Distributed turbo coded diversity for relay channel," Electronics Letters, vol. 39, pp. 786-787, May 2003.

[8] L. Lampe, R. Schober, V. Pauli, and C. Windpassinger, "Multiple-symbol differential sphere decoding," IEEE Transactions on Communications, vol. 12, pp. 1981-1985, Dec. 2005.

[9] J. G. Proakis, Digital Communications. 4th edition, New York, NY: Mc-Graw-Hill, 2000.

[10] L. Wang, L. Kong, S. X. Ng, and L. Hanzo, "To cooperate or not: A capacity perspective," in submitted to 2010 IEEE 71th Vehicular Technology Conference (VTC 2010-Spring), http://eprints.ecs.soton.ac.uk/18049/1/near_capcity_single_relay.pdf.

[11] R.-R. Chen, R. Koetter, and U. Madhow, "Joint noncoherent demodulation and decoding for fast rayleigh fading channels," Conference on Information Sciences and Systems, Mar. 2003.

[12] V. Pauli, L. Lampe, and R. Schober, "“Turbo DPSK” using soft multiplesymbol differential sphere decoding," IEEE Transactions on Information Theory, vol. 52, no. 4, pp. 1385-1398, 2006.

[13] A. Ashikhmin, G. Kramer, and S. ten Brink, "Extrinsic information transfer functions: model and erasure channel properties," IEEE Transactions on Information Theory, vol. 50, pp. 2657-2673, Nov. 2004.

[14] J. Kliewer, A. Huebner, and D. J. Costello, "On the achievable extrinsic information of inner decoders in serial concatenation," in Information Theory, 2006 IEEE International Symposium on, (Seattle, WA), pp. 2680-2684, July 2006.

[15] M. Tüchler, "Design of serially concatenated systems depending on the block length,' IEEE Transactions on Communications, vol. 52, pp. 209218, Feb. 2004 